\title{
The Willmore Conjecture for immersed tori with small curvature integral
}

\author{
Bernd Ammann
}

June 1999

\begin{abstract}
The Willmore conjecture states that any immersion $F: T^{2} \rightarrow \mathbb{R}^{n}$ of a 2-torus into euclidean space satisfies $\int_{T^{2}} H^{2} \geq 2 \pi^{2}$. We prove it under the condition that the $L^{p}$-norm of the Gaussian curvature is sufficiently small.
\end{abstract}

Keywords: Willmore integral, conformal metrics, two-dimensional torus

Mathematics Classification: 53A05 (Primary), 53A30, 58G30 (Secondary)

\section{Introduction}

Let $F: N \rightarrow \mathbb{R}^{n}$ be an immersion of a closed surface $N$ into Euclidean space. The Willmore integral $\mathcal{W}(F)$ is defined using the mean curvature $H$ of the immersion $F$ and the area form darea associated to the induced metric:

$$
\mathcal{W}(F):=\int_{N} H^{2} \text { darea. }
$$

In the case $n=3$ we easily get a lower estimate. If $\kappa_{1}$ and $\kappa_{2}$ are the principal curvatures we have $H^{2}=\left(\kappa_{1}+\kappa_{2}\right)^{2} / 4 \geq \max \left\{0, \kappa_{1} \kappa_{2}\right\}$. But $\kappa_{1} \kappa_{2}$ is just the Gaussian curvature $K$ of $\left(N, F^{*} g_{\text {eucl }}\right)$ which is equal to the determinant of the Gauss map $N \rightarrow S^{2}$. Thus we obtain

$$
\mathcal{W}(F) \geq \int_{N^{+}} K \text { darea } \geq \int_{S^{2}} 1 \text { darea }=4 \pi,
$$

with $N^{+}=\{x \in N \mid K(x) \geq 0\}$ which is mapped onto $S^{2}$ via the Gauss map.

The value $4 \pi$ is attained if $F: S^{2} \rightarrow \mathbb{R}^{3}$ is the standard embedding. And vice versa if $\mathcal{W}(F)=4 \pi$ we know that $N$ is $S^{2}$ whose image $F\left(S^{2}\right)$ is a round sphere. 
This well-known result has been improved and generalized by Li and Yau LY82, Fact 3]. For arbitrary dimension $n \geq 3$ we assume that $F^{-1}(p)$ contains $k$ points for some $p \in \mathbb{R}^{n}$. Then Li and Yau showed the estimate

$$
\mathcal{W}(F) \geq 4 \pi k
$$

If $N$ has positive genus, then the value $4 \pi$ will never be attained. In this paper we will study the following conjecture attributed to Willmore Wil65.

CONJECTURE 1.1 (Willmore conjecture). For any immersion $F: T^{2} \rightarrow \mathbb{R}^{n}$ of the 2-dimensional torus into $\mathbb{R}^{n}, n \geq 3$, the inequality

$$
\mathcal{W}(F) \geq 2 \pi^{2}
$$

holds.

Leon Simon proved in Sim93 that for any fixed dimension $n \geq 3$ the infimum

$$
\inf \left\{\mathcal{W}(F) \mid F: T^{2} \rightarrow \mathbb{R}^{n}\right\}
$$

is actually attained and he concludes that there is an estimate $W(F) \geq 4 \pi+\varepsilon_{n}$ with $\varepsilon_{n}>0$ for any $n \geq 3$ without giving an explicit value for $\varepsilon_{n}$. But the Willmore conjecture remains open until today.

Nevertheless in many special cases the Willmore conjecture has been confirmed.

If $n=3$ and if the image $F\left(T^{2}\right)$ has a rotational symmetry, the Willmore conjecture has been proven by Langer and Singer in [LS84. Shiohama and Takagi ST70 and independently Willmore Wil71 showed that the Willmore conjecture is true if $F\left(T^{2}\right)$ is the boundary of an $\varepsilon$-neighborhood of a closed curve in $\mathbb{R}^{3}$ with $\varepsilon$ sufficiently small.

It should be mentioned here that there is a natural generalization of the Willmore functional to immersions $F$ of a closed surface $N$ into a Riemannian manifold $(M, h)$ by defining

$$
\mathcal{W}(F):=\int_{N}\left(H^{2}+K_{M}\right) \text { darea, }
$$

where $K_{M}(p)$ is the sectional curvature of $(M, h)$ evaluated at the plane $d F\left(T_{p} N\right)$. This functional is invariant under conformal changes of $h$ Tho23, Wei78 . Hence, the Willmore conjecture for immersions $T^{2} \rightarrow \mathbb{R}^{n}$ is equivalent to the Willmore conjecture for immersions $F: T^{2} \rightarrow S^{n} \subset \mathbb{R}^{n+1}$. The Willmore conjecture for immersions of the latter kind has been proven by Ros [Ros97] under the additional condition that $n=3$ and that $F\left(T^{2}\right)$ is invariant under the antipodal map of $S^{3}$.

Other partial solutions to the Willmore conjecture use spectral geometry. If $F$ : $(N, g) \rightarrow S^{n}$ or equivalently $F:(N, g) \rightarrow \mathbb{R}^{n}$ is a conformal immersion of a closed 
surface $N$ and if $\lambda_{1}$ is the first positive eigenvalue of the Laplace operator on $(N, g)$, then Li and Yau [LY82, Theorem 1] proved

$$
W(F) \geq \frac{1}{2} \lambda_{1} \operatorname{area}(N, g)
$$

Every Riemannian 2-torus is conformally equivalent to a flat one, say $\left(\mathbb{R}^{2} / \Gamma_{x y}, g_{\text {eucl }}\right)$ where the lattice $\Gamma_{x y}$ is generated by $(1,0)$ and $(x, y), 0 \leq x \leq 1 / 2, x^{2}+y^{2} \geq 1$, $y>0$ and where $g_{\text {eucl }}$ is the Euclidean standard metric on $\mathbb{R}^{2}$. The first positve eigenvalue of the Laplace operator of $\left(\mathbb{R}^{2} / \Gamma_{x y}, g_{\text {eucl }}\right)$ is $4 \pi^{2} / y^{2}$ and the area is $y$. Thus Li and Yau get the corollary

$$
W(F) \geq \frac{2 \pi^{2}}{y}
$$

which proves the Willmore conjecture for $y \leq 1$, i. e. for a subset of the moduli space that has positive measure (see figure 11). The set of conformal equivalence classes for which we know the Willmore conjecture has been enlarged by Montiel and Ros MR85. They proved that $y \leq 1$ could be replaced by the weaker condition

$$
\left(x-\frac{1}{2}\right)^{2}+(y-1)^{2} \leq \frac{1}{4} .
$$

Replacing the Laplace operator by the Dirac operator there is a similar result. If $F:(N, g) \rightarrow S^{n}$ or $F:(N, g) \rightarrow \mathbb{R}^{n}$ is an isometric immersion, then Bär Bär98 established the estimate

$$
W(F) \geq \mu_{1} \operatorname{area}\left(T^{2}, g\right)
$$

where $\mu_{1}$ is the first eigenvalue of a twisted Dirac operator on $T^{2}$. Unfortunately this statement is no longer true if we replace "isometric immersion" by "conformal immersion", so in order to apply this estimate we have to get lower bounds for eigenvalues of Dirac operators on non-flat 2-tori. Such bounds have been found by the author in [Amm98] or alternatively Amm. With these estimates we proved the Willmore conjecture in an open subset of the spin-conformal moduli space provided that the $L^{p}$-norm of the Gaussian curvature of $N$ is sufficiently small. This open subset is disjoint from the subset in which Montiel and Ros proved the Willmore conjecture.

A special situation is given if the immersion $F: T^{2} \rightarrow \mathbb{R}^{n}$ is flat, i. e. the induced metric on $F\left(T^{2}\right)$ has vanishing Gaussian curvature. Examples of such tori are the Clifford torus

$$
\frac{1}{\sqrt{2}} S^{1} \times \frac{1}{\sqrt{2}} \subset S^{3}
$$

or the Hopf tori Pin85. For flat immersions the Willmore conjecture is true Che81. 


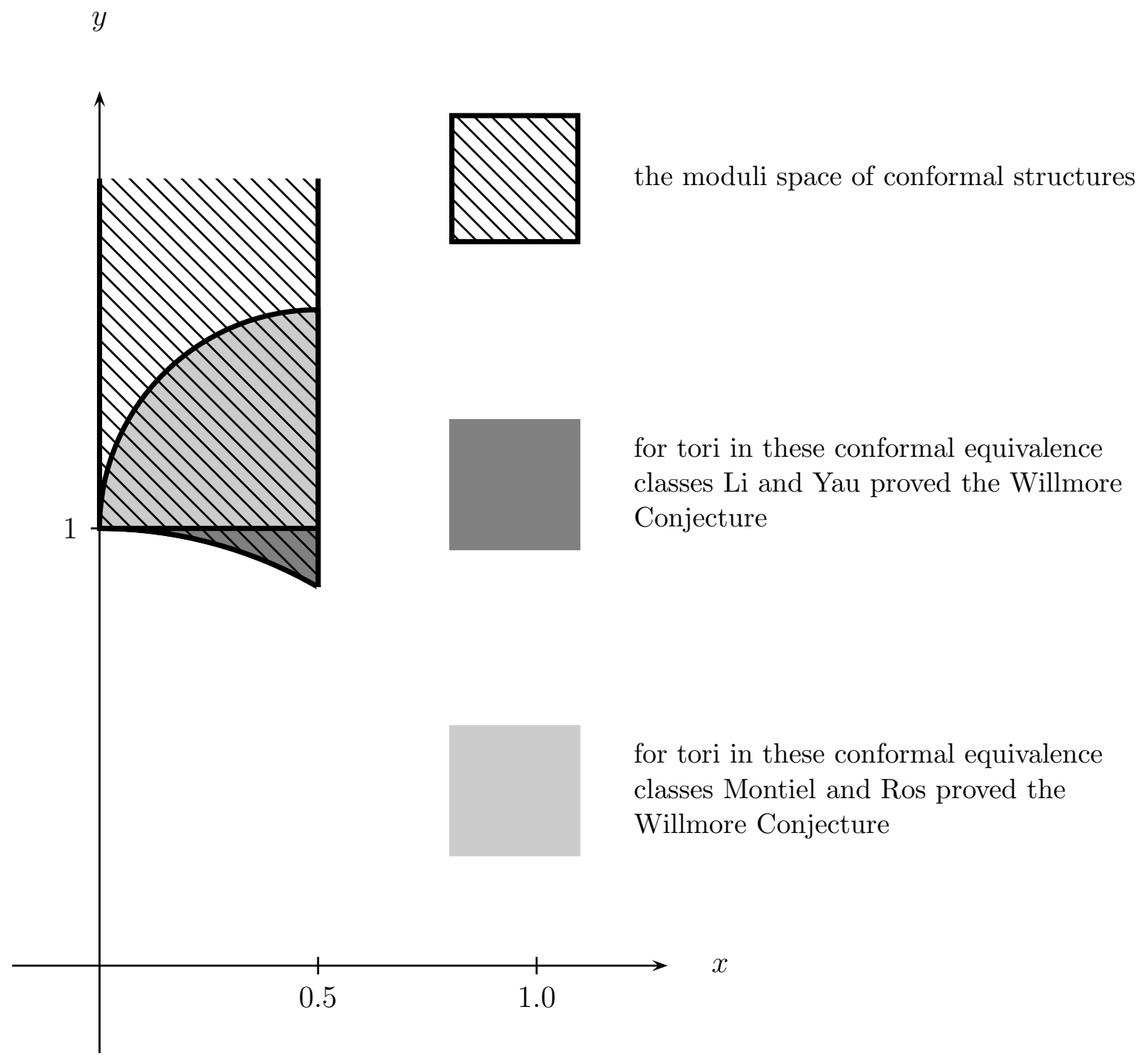

Figure 1: The moduli space of conformal structures on $T^{2}$ 
Topping Top98 gave a proof of the Willmore conjecture for non-flat immersions $F: T^{2} \rightarrow S^{3} \subset \mathbb{R}^{4}$ if the following condition is satisfied

$$
\int_{T^{2}}\left|K_{g}\right| \text { darea }_{g} \leq \frac{2}{\pi}\left(W(F)-\operatorname{area}\left(T^{2}, g\right)\right),
$$

where $g$ is the induced metric. Note that the left hand side is the $L^{1}$-norm of the Gaussian curvature and the right hand side can be rewritten as

$$
\frac{2}{\pi} \int_{T^{2}}\left(H_{T^{2} \rightarrow S^{3}}\right)^{2} \text { darea }_{g}
$$

where $H_{T^{2} \rightarrow S^{3}}$ is the relative mean curvature of $T^{2}$ lying in $S^{3}$.

The main theorems of this article now prove the Willmore conjecture under a similar condition on the Gaussian curvature, although our methods are completely different. Here we assume that the $L^{p}$-norm, $p>1$ of the Gaussian curvature is bounded by functions that only depend on intrinsic invariants of $\left(T^{2}, g\right)$. So our assumptions are - in contrast to Topping's results - purely intrinsic in the following sense: we construct many non-flat Riemannian metrics $g$ on $T^{2}$ such that any isometric immersion $F:\left(T^{2}, g\right) \rightarrow\left(\mathbb{R}^{n}, g_{\text {eucl }}\right)$ satisfies $W(F) \geq 2 \pi^{2}$.

MAIN THEOREM I. For any real number $p>1$ and any conformal equivalence class $c$ on $T^{2}$ there exists $\tau(c, p)>0$ such that the following holds:

If $F: T^{2} \rightarrow \mathbb{R}^{n}$ is an immersion with induced metric $g:=F^{*} g_{\mathrm{eucl}}$ and conformal equivalence class $[g]$ satisfying

$$
\left\|K_{g}\right\|_{L^{p}\left(T^{2}, g\right)} \cdot\left(\operatorname{area}\left(T^{2}, g\right)\right)^{1-\frac{1}{p}}<\tau([g], p),
$$

then the Willmore conjecture

$$
W(F) \geq 2 \pi^{2}
$$

holds.

MAIN THEOREM II. There exists a function $\rho:] 0, \infty[\times] 0, \infty[\times] 1, \infty[\rightarrow] 0, \infty[$ with the following property:

If $F: T^{2} \rightarrow \mathbb{R}^{n}$ is an immersion whose induced metric $g:=F^{*} g_{\mathrm{eucl}}$ satisfies

$$
\left\|K_{g}\right\|_{L^{p}\left(T^{2}, g\right)}<\rho\left(\operatorname{area}\left(T^{2}, g\right), \operatorname{sys}_{1}\left(T^{2}, g\right), p\right)
$$

for some $p>1$, then the Willmore conjecture

$$
W(F) \geq 2 \pi^{2}
$$

holds. 
These results generalize most of the statements about the Willmore conjecture in Amm98. The methods used in the proof are strongly related. The estimate of section 3 can also be used to get spectral estimates on 2-dimensional tori. This application will be presented in another article Amm which is in preparation.

Acknowledgement. I want to thank Christian Bär, Ernst Kuwert and Reiner Schätzle for many interesting discussions about the subject.

\section{Lower bounds for the Willmore functional}

In this section we will prove the main theorems of the article using Theorem 3.1 which will be shown in section 3 .

At first we will define some geometric quantities of Riemannian 2-tori $\left(T^{2}, g\right)$. Let $K_{g}$ be the Gaussian curvature, $K_{g}^{+}:=\max \left\{K_{g}, 0\right\}$ and $K_{g}^{-}:=\min \left\{K_{g}, 0\right\}$. For $p \in[1, \infty[$ we set

$$
\begin{aligned}
\mathcal{K}_{p}(g) & :=\left\|K_{g}\right\|_{L^{p}\left(T^{2}, g\right)} \cdot\left(\operatorname{area}\left(T^{2}, g\right)\right)^{1-\frac{1}{p}} \\
\mathcal{K}_{p}^{ \pm}(g) & :=\left\|K_{g}^{ \pm}\right\|_{L^{p}\left(T^{2}, g\right)} \cdot\left(\operatorname{area}\left(T^{2}, g\right)\right)^{1-\frac{1}{p}} .
\end{aligned}
$$

The Hölder inequality yields $\mathcal{K}_{p}(g) \leq \mathcal{K}_{p^{\prime}}(g)$ and $\mathcal{K}_{p}^{ \pm}(g) \leq \mathcal{K}_{p^{\prime}}^{ \pm}(g)$ for $p \leq p^{\prime}$. Note that these quantities are invariant under rescaling of the metric.

The 1-systole or simply the systole $\operatorname{sys}_{1}\left(T^{2}, g\right)$ is the length of the shortest noncontracible loop in $T^{2}$. We define the geometric quantity

$$
\mathcal{V}\left(T^{2}, g\right):=\frac{\operatorname{area}\left(T^{2}, g\right)}{\operatorname{sys}_{1}\left(T^{2}, g\right)^{2}}
$$

which is also invariant under rescaling of the metric. Furthermore we set

$$
\mathcal{M}:=\left\{(x, y) \in \mathbb{R}^{2} \mid 0 \leq x \leq 1 / 2, x^{2}+y^{2} \geq 1, y \geq 0\right\}
$$

As in the introduction we define for $(x, y) \in \mathcal{M}$ the lattice $\Gamma_{x y}$ in $\mathbb{R}^{2}$ to be generated by $(1,0)$ and $(x, y)$. Unless otherwise stated $\mathbb{R}^{2} / \Gamma_{x y}$ always carries the Riemannian metric induced by the Euclidean metric of $\mathbb{R}^{2}$. Every Riemannian metric on $T^{2}$ is conformally equivalent to exactly one torus of the form $\mathbb{R}^{2} / \Gamma_{x y}$ with $(x, y) \in \mathcal{M}$. Hence $\mathcal{M}$ can be identified with the moduli space of conformal structures on $T^{2}$. With these notations we have

$$
y=\mathcal{V}\left(\mathbb{R}^{2} / \Gamma_{x y}\right)
$$


The oscillation of a continuous function $u: T^{2} \rightarrow \mathbb{R}$ is defined to be osc $u:=$ $\max u-\min u$ where the maximum and minimum is to be taken over $T^{2}$.

Now, we will cite a lemma.

LEMMA 2.1 ([Bes87, 1.159]). Let $g_{1}$ and $g_{2}=e^{2 u} g_{1}$ be two conformal Riemannian metrics on a surface. Then their Gaussian curvatures are related via

$$
K_{g_{2}}-e^{-2 u} K_{g_{1}}=\Delta_{g_{2}} u=e^{-2 u} \Delta_{g_{1}} u .
$$

We always use the convention $\Delta=-* d * d$, i. e. $\Delta$ has nonnegative eigenvalues on compact sets with Dirichlet boundary conditions.

The formula of the lemma also yields a simple proof of the uniformisation theorem for $T^{2}$ stating that any Riemannian metric on $T^{2}$ is conformally equivalent to a flat one - a fact that has already been used several times in this article.

LEMMA 2.2. Suppose that $T^{2}$ carries a flat metric $g_{0}$ and another metric $g$ conformal to $g_{0}$. Then

$$
\mathcal{V}\left(T^{2}, g\right) \geq \mathcal{V}\left(T^{2}, g_{0}\right)
$$

Proof. The proof of this lemma follows a proof of Loewner's theorem, see e.g. [Gro81, 4.1].

We write $g=e^{2 u} g_{0}$. Obviously, area $\left(T^{2}, g\right)=\int_{T^{2}} e^{2 u}$ darea $_{g_{0}}$ and area $\left(T^{2}, g_{0}\right)=$ $\int_{T^{2}}$ darea $_{g_{0}}$. Let $c: S^{1} \rightarrow T^{2}$ be a non contractible loop of minimal length $l_{0}:=$ $\operatorname{sys}_{1}\left(T^{2}, g_{0}\right)$ with respect to $g_{0}$. Then for $a \in T^{2}$ the translated loop $c_{a}(\cdot):=c(\cdot)+a$ has the same length with respect to $g_{0}$. Let $l(a)$ be the length of $c_{a}$ with respect to $g$. Then

$$
\begin{aligned}
\int_{T^{2}} l(a) \text { darea }_{g_{0}} & =\int_{T^{2}} \text { darea }_{g_{0}} \int_{S^{1}} d t\left|\dot{c}_{a}(t)\right|_{g} \\
& =\int_{T^{2}} \text { darea }_{g_{0}} \int_{S^{1}} d t e^{u \circ c_{a}(t)}\left|\dot{c}_{a}(t)\right|_{g_{0}} \\
& =l_{0} \int_{T^{2}} e^{u} \text { darea }_{g_{0}} \\
& \leq l_{0} \operatorname{area}\left(T^{2}, g_{0}\right)^{1 / 2} \operatorname{area}\left(T^{2}, g\right)^{1 / 2}
\end{aligned}
$$

So there is a point $a \in T^{2}$ with

$$
\frac{\operatorname{sys}_{1}\left(T^{2}, g\right)^{2}}{\operatorname{area}\left(T^{2}, g\right)} \leq \frac{l(a)^{2}}{\operatorname{area}\left(T^{2}, g\right)} \leq \frac{l_{0}^{2}}{\operatorname{area}\left(T^{2}, g_{0}\right)}=\frac{\operatorname{sys}_{1}\left(T^{2}, g_{0}\right)^{2}}{\operatorname{area}\left(T^{2}, g_{0}\right)}
$$

Using the fact that $\mathcal{V}\left(T^{2}, g_{0}\right) \geq \sqrt{3} / 2$ for any flat metric $g_{0}$ we get the 
COROLlARY 2.3 (Loewner's theorem). For any Riemannian 2-torus $\left(T^{2}, g\right)$ we have

$$
\mathcal{V}\left(T^{2}, g\right) \geq \frac{\sqrt{3}}{2}
$$

Equality is attained only for the equilateral flat torus.

COROLlaRY 2.4. Any isometric immersion $F:\left(T^{2}, g\right) \rightarrow\left(\mathbb{R}^{n}, g_{\text {eucl }}\right)$ satisfies

$$
W(F) \geq \frac{2 \pi^{2}}{\mathcal{V}\left(T^{2}, g\right)}=\frac{2 \pi^{2} \operatorname{sys}_{1}\left(T^{2}, g\right)^{2}}{\operatorname{area}\left(T^{2}, g\right)} .
$$

In particular, the Willmore conjecture is satisfied for any isometrically immersed torus with $\operatorname{sys}_{1}\left(T^{2}, g\right)^{2} \geq \operatorname{area}\left(T^{2}, g\right)$.

Proof. We write $g=e^{2 u} g_{0}$ with $g_{0}$ flat. Let $\left(T^{2}, g_{0}\right)$ be isometric to $\mathbb{R}^{2} / \Gamma_{x y}$ with $x, y \in \mathcal{M}$. Then

$$
y=\mathcal{V}\left(T^{2}, g_{0}\right) \leq \mathcal{V}\left(T^{2}, g\right) .
$$

Now the corollary follows from inequality (1) of the introduction.

The following lemma is a converse to Lemma 2.2.

LEMMA 2.5. Suppose that $T^{2}$ carries a flat metric $g_{0}$ and another metric $g=e^{2 u} g_{0}$. Then

$$
\mathcal{V}\left(T^{2}, g\right) \leq e^{2 \operatorname{osc} u} \mathcal{V}\left(T^{2}, g_{0}\right) .
$$

The proof is straightforward.

We will prove our main theorems in a slightly stronger version than stated in the introduction.

MAIN THEOREM I. There exists a function $\tau:[\sqrt{3} / 2, \infty[\times] 1, \infty[\rightarrow] 0, \infty[$ with the following property:

If $F: T^{2} \rightarrow \mathbb{R}^{n}$ is an immersion such that the induced metric $g:=F^{*} g_{\mathrm{eucl}}$ satisfies

$$
\mathcal{K}_{p}(g)<\tau(y, p) \quad \text { and } \quad\left(T^{2}, g\right) \text { is conformally equivalent to } \mathbb{R}^{2} / \Gamma_{x y}
$$

for some $p>1$, then the Willmore conjecture

$$
W(F) \geq 2 \pi^{2}
$$

holds. 
Example. Let $F:\left(T^{2}, g_{0}\right) \rightarrow\left(\mathbb{R}^{n}, g_{\text {eucl }}\right)$ be a conformal immersion. Suppose that $\left(T^{2}, g_{0}\right)$ is isometric to $\left(\mathbb{R}^{2} / \Gamma_{x 2}, g_{\text {eucl }}\right), 0 \leq x \leq 1 / 2$. From the explicit construction of $\tau$ in the proof we see that the Willmore conjecture is satisfied if

$$
\|K\|_{L^{2}\left(T^{2}, g\right)} \operatorname{area}\left(F\left(T^{2}\right)\right)^{1 / 2} \leq 0.1987553 .
$$

Remark. The $\tau$ constructed in the proof is continuous on $[\sqrt{3} / 2,1[\times] 1, \infty[$ and on ] $1, \infty[\times] 1, \infty[$, but

$$
\lim _{y \searrow 1} \tau(y, p)=0 \neq \tau(1, p)
$$

for any $p \in] 1, \infty[$. Hence $\tau$ is not continuous at $(y, p)$ with $y=1$. Nevertheless, if we view $\tau$ as a function on $\mathcal{M} \times] 1, \infty[$ we can combine Main Theorem I with the result of Montiel and Ros mentioned in the introduction MR85 to get a similar function $\widetilde{\tau}: \mathcal{M} \times] 1, \infty\left[\rightarrow \mathbb{R}^{+}\right.$that is continuous on $\left.\left(\mathcal{M}-\left\{\left[\mathbb{R}^{2} / \Gamma_{01}\right]\right\}\right) \times\right] 1, \infty[$ and such that Main Theorem I holds with $\tau$ replaced by $\widetilde{\tau}$.

MAIN THEOREM II. There exists a function $\sigma:[\sqrt{3} / 2, \infty[\times] 1, \infty[\rightarrow] 0, \infty[$ with the following property:

If $F: T^{2} \rightarrow \mathbb{R}^{n}$ is an immersion such that the induced metric $g:=F^{*} g_{\text {eucl }}$ satisfies

$$
\mathcal{K}_{p}(g)<\sigma\left(\mathcal{V}\left(T^{2}, g\right), p\right)
$$

for some $p>1$, then the Willmore conjecture

$$
W(F) \geq 2 \pi^{2}
$$

holds.

Remark. In analogy to the previous remark, we cannot chose $\sigma$ to be continuous at $\mathcal{V}=1, p$ arbitrary. But $\sigma$ can be chosen to be continuous on $\{(\mathcal{V}, p) \mid \mathcal{V} \neq 1\}$.

A central role in the proof is played by

LEMMA 2.6. Let $F: T^{2} \rightarrow \mathbb{R}^{n}$ be an immersion. Let $g_{0}$ denote the standard metric on $\mathbb{R}^{2}$ and suppose that $T^{2}$ with the induced metric $F^{*} g_{\text {eucl }}$ is isometric to $\left(\mathbb{R}^{2} / \Gamma_{x y}, e^{2 u} g_{0}\right),(x, y) \in \mathcal{M}$, where $u$ is a smooth function. Then

$$
W(F) \geq e^{-2 \operatorname{osc} u} \pi^{2}\left(y+\frac{1}{y}\right)
$$

This lemma will be shown at the end of this section.

We now define

$$
\begin{aligned}
\mathcal{Q}(\mathcal{K}, p, \mathcal{V}):=\exp \quad\left[\left|\log \left(1-\frac{\mathcal{K}}{4 \pi}\right)\right|+\frac{\mathcal{K}}{4 \pi-\mathcal{K}} q \log (2 q)\right. \\
\left.+\frac{q \mathcal{K}}{2 \pi}+\frac{\mathcal{K} \mathcal{V}}{4}\right]
\end{aligned}
$$


with $q:=p /(p-1)$. From the previous lemma and from Theorem 3.1 we get a corollary.

COROLLARY 2.7. Under the conditions of the previous lemma we have

$$
\begin{aligned}
& W(F) \geq \mathcal{Q}\left(\mathcal{K}_{p}(g), p, \mathcal{V}\left(T^{2}, g\right)\right)^{-1} \pi^{2}\left(y+\frac{1}{y}\right) \\
& W(F) \geq \mathcal{Q}\left(\mathcal{K}_{p}(g), p, y\right)^{-1} \pi^{2}\left(y+\frac{1}{y}\right) .
\end{aligned}
$$

if $\mathcal{K}_{p}(g)<4 \pi$.

Proof of Main Theorem I. Let $\left(T^{2}, g\right)$ be conformally equivalent to $\mathbb{R}^{2} / \Gamma_{x y}$, $(x, y) \in \mathcal{M}$. We distinguish between two cases.

In the case $y \leq 1$ we can use the result of Li-Yau [LY82 or Montiel-Ros MR85 to see that $\tau(y, p)$ can be chosen as any arbitrary positive real number.

On the other hand, for $y>1$ we get

$$
y+\frac{1}{y}>2 .
$$

The function $\mathcal{Q}(\mathcal{K}, p, \mathcal{V})$ is continuous and for $\mathcal{K} \rightarrow 0$ with $p$ and $\mathcal{V}$ fixed $\mathcal{Q}$ converges to 1 . Therefore there is a real number $\tau(y, p)>0$ such that

$$
\mathcal{Q}(\mathcal{K}, p, y)^{-1}\left(y+\frac{1}{y}\right)>2
$$

whenever $\mathcal{K} \leq \tau(y, p)$.

Obviously $\tau$ can be chosen as a continuous function on $] 1, \infty[\times] 1, \infty[$.

Proof of Main Theorem II. As in the proof of Main Theorem I we will distinguish between two cases for the construction of $\sigma(\mathcal{V}, p)$.

In the first case, $\mathcal{V} \leq 1$, according to Corollary 2.4 the Willmore conjecture is satisfied for any immersion $F: T^{2} \rightarrow \mathbb{R}^{n}$ with $\mathcal{V}\left(T^{2}, F^{*} g_{\text {eucl }}\right)=\mathcal{V}$, hence Main Theorem II is true if $\sigma(\mathcal{V}, p)$ is any positive number.

In the remaining case, i. e. $\mathcal{V}>1$, we can choose $\sigma_{1}(\mathcal{V}, p)>0$ such that

$$
\mathcal{Q}(\mathcal{K}, p, \mathcal{V}) \leq \sqrt{\mathcal{V}}
$$

whenever $\mathcal{K} \in\left[0, \sigma_{1}(\mathcal{V}, p)\right]$. So let $g=e^{2 u} g_{0}$ be an arbitrary metric on $T^{2}$ with $g_{0}$ flat and suppose $\mathcal{K}_{p}(g) \leq \sigma_{1}\left(\mathcal{V}\left(T^{2}, g\right), p\right)$, then according to Theorem 3.1

$$
e^{2 \text { osc } u} \leq \mathcal{Q}\left(\mathcal{K}_{p}(g), p, \mathcal{V}\left(T^{2}, g\right)\right) \leq \sqrt{\mathcal{V}\left(T^{2}, g\right)} .
$$


Using Lemma 2.5 we get

$$
\mathcal{V}\left(T^{2}, g_{0}\right) \geq \sqrt{\mathcal{V}\left(T^{2}, g\right)}>1 .
$$

Let $\left(T^{2}, g\right)$ be conformally equivalent to $\mathbb{R}^{2} / \Gamma_{x y}$ with $(x, y) \in \mathcal{M}$. Then the above inequality and Lemma 2.2 yield $\sqrt{\mathcal{V}\left(T^{2}, g\right)} \leq y \leq \mathcal{V}\left(T^{2}, g\right)$.

Now set

$$
\sigma(\mathcal{V}, p):=\min \left\{\sigma_{1}(\mathcal{V}, p), \min \{\tau(v, p) \mid \sqrt{\mathcal{V}} \leq v \leq \mathcal{V}\}\right\}>0
$$

with the function $\tau$ constructed in the proof of Main Theorem I. Because of the construction of $\sigma$ we get $\sigma\left(\mathcal{V}\left(T^{2}, g\right), p\right) \leq \tau(y, p)$. Therefore Main Theorem II follows from Main Theorem I. The continuity property is clear from the construction of $\sigma$.

Proof of Lemma 2.6. The lemma is a generalization of [LY82, Prop. 2, page 287]. We will adapt the proof of this proposition to our situation by introducing conformal factors into the formulas.

Let $g:=F^{*} g_{\text {eucl }}$ be the metric on $T^{2}$ induced by the Euclidean metric on $\mathbb{R}^{n}$. We decompose $F$ into its coordinate functions $F=\left(F_{1}, \ldots, F_{n}\right), F_{i}: T^{2} \rightarrow \mathbb{R}$.

We get

$$
\begin{aligned}
4 W(F) & =4 \int_{T^{2}}|H|^{2} \text { darea }_{g} \\
& =\sum_{i=1}^{n} \int_{T^{2}}\left(\Delta_{g} F_{i}\right)^{2} \text { darea }_{g} \\
& =\sum_{i=1}^{n} \int_{T^{2}} e^{-2 u}\left(\Delta_{g_{0}} F_{i}\right)^{2} \text { darea }_{g_{0}} \\
& \geq \sum_{i=1}^{n} \int_{T^{2}} e^{-2 \max u}\left(\Delta_{g_{0}} F_{i}\right)^{2} \text { darea }_{g_{0}} .
\end{aligned}
$$

After a translation we can assume

$$
\int_{T^{2}} F_{i} \text { darea }_{g_{0}}=0 \quad \text { for any } i=1, \ldots, n .
$$

Now we make a Fourier decomposition for the functions $F_{i}$ :

$$
\begin{aligned}
F_{i}(w)= & \sum_{\substack{p, q \in \mathbb{Z} \\
(p, q) \neq(0,0)}} A_{i p q} \sqrt{\frac{2}{y}} \cos \left(2 \pi\left\langle\left(q, \frac{p-q x}{y}\right), w\right\rangle\right) \\
& +\sum_{\substack{p, q \\
(p, q) \neq(0,0)}} B_{i p q} \sqrt{\frac{2}{y}} \sin \left(2 \pi\left\langle\left(q, \frac{p-q x}{y}\right), w\right\rangle\right) .
\end{aligned}
$$


This means for our estimate of $W(F)$

$$
\begin{aligned}
4 W(F) \geq & e^{-2 \max u} 16 \pi^{4}\left[\sum_{\substack{i, p, q \\
(p, q) \neq(0,0)}} A_{i p q}^{2}\left(q^{2}+\left(\frac{p-q x}{y}\right)^{2}\right)^{2}\right. \\
& \left.+\sum_{\substack{i, p, q \\
(p, q) \neq(0,0)}} B_{i p q}^{2}\left(q^{2}+\left(\frac{p-q x}{y}\right)^{2}\right)^{2}\right] \\
\geq & e^{-2 \max u} 16 \pi^{4}\left[\sum_{\substack{i, p, q \\
(p, q) \neq(0,0)}}\left(A_{i p q}^{2}+B_{i p q}^{2}\right)\left(q^{2}+\frac{1}{y^{2}}\left(\frac{p-q x}{y}\right)^{2}\right)\right] .
\end{aligned}
$$

For the last estimate we used that the inequality

$$
\left[q^{2}+\left(\frac{p-q x}{y}\right)^{2}\right]^{2} \geq q^{2}+\left[2 q^{2}+\left(\frac{p-q x}{y}\right)^{2}\right]\left(\frac{p-q x}{y}\right)^{2} \geq q^{2}+\frac{1}{y^{2}}\left(\frac{p-q x}{y}\right)^{2}
$$

holds for any $(p, q) \in \mathbb{Z} \times \mathbb{Z}$ with $(p, q) \neq(0,0)$ and $x, y \in \mathbb{R}$ with $2 y^{2} \geq 1$.

Now we transform the right hand side of inequality (21). The projections of the vectors $(1,0),(0,1)$ of $\mathbb{R}^{2}$ to the quotient $T^{2}=\mathbb{R}^{2} /\langle(1,0),(x, y)\rangle$ define two vector fields denoted $e_{1}$ and $e_{2}$. These vector fields form an orthonormal frame for the metric $g_{0}$.

The conformal factor $e^{2 u}$ can be calculated as

$$
e^{2 u}=\left|\partial_{e_{1}} F\right|_{g_{\text {eucl }}}^{2}=\sum_{i}\left(\partial_{e_{1}} F_{i}\right)^{2}
$$

Therefore we get

$$
\operatorname{area}\left(T^{2}, g\right)=\int e^{2 u} \text { darea }_{g_{0}}=\sum_{i} \int_{T^{2}}\left(\partial_{e_{1}} F_{i}\right)^{2} \text { darea }_{g_{0}}=4 \pi^{2} \sum_{\substack{i, p, q \\(p, q) \neq(0,0)}}\left(A_{i p q}^{2}+B_{i p q}^{2}\right) q^{2} \text {. }
$$

If we replace $e_{1}$ by $e_{2}$ we get completely analogously

$$
\operatorname{area}\left(T^{2}, g\right)=4 \pi^{2} \sum_{\substack{i, p, q \\(p, q) \neq(0,0)}}\left(A_{i p q}^{2}+B_{i p q}^{2}\right)\left(\frac{p-q x}{y}\right)^{2} \text {. }
$$

On the other hand

$$
\operatorname{area}\left(T^{2}, g\right)=\int e^{2 u} \text { darea }_{g_{0}} \geq e^{2 \min u} \operatorname{area}\left(T^{2}, g_{0}\right)=e^{2 \min u} \cdot y \text {. }
$$

All these inequalities together imply

$$
W(F) \geq e^{-2 \max u} \pi^{2}\left(1+\frac{1}{y^{2}}\right) \operatorname{area}\left(T^{2}, g\right) \geq e^{-2 \operatorname{osc} u} \pi^{2}\left(y+\frac{1}{y}\right) .
$$




\section{Controling the conformal scaling function}

Consider the 2-dimensional torus with a Riemannian metric $g$. As in the previous section we write this metric in the form

$$
g=e^{2 u} g_{0}
$$

where $g_{0}$ is a flat metric. The function $u$ is uniquely determined by $g$ up to an additive constant. The aim of this section is to prove estimates for the oscillation osc $u=\max u-\min u$. We will give such an estimate for the case $\mathcal{K}_{1}(g)=$ $\int_{T^{2}}\left|K_{g}\right|$ darea $_{g}<4 \pi$. In this situation we can choose $p>1$ such that $\mathcal{K}_{p}(g)<4 \pi$. In order to motivate the condition $\mathcal{K}_{p}(g)<4 \pi$ we construct some examples in section 5. If $\mathcal{K}_{p}(g)$ is sufficiently big, then there is a bubbling phenomenon as described for example in Che98 for the special case $p=2$.

THEOREM 3.1. We assume for some $p \in] 1, \infty[$

$$
\mathcal{K}_{p}:=\mathcal{K}_{p}\left(T^{2}, g\right)<4 \pi
$$

Then the oscillation of $u$ is bounded as follows

$$
\begin{gathered}
\operatorname{osc} u \leq \mathcal{S}\left(\mathcal{K}_{p}, p, \mathcal{V}\left(T^{2}, g_{0}\right)\right), \\
\text { osc } u \leq \mathcal{S}\left(\mathcal{K}_{p}, p, \mathcal{V}\left(T^{2}, g\right)\right),
\end{gathered}
$$

where we use the definition

$$
\mathcal{S}(\mathcal{K}, p, \mathcal{V}):=\frac{1}{2}\left|\log \left(1-\frac{\mathcal{K}}{4 \pi}\right)\right|+\frac{\mathcal{K}}{8 \pi-2 \mathcal{K}} q \log (2 q)+\frac{q \mathcal{K}}{4 \pi}+\frac{\mathcal{K} \mathcal{V}}{8}
$$

with $q:=p /(p-1)$.

\section{Remark.}

$$
\mathcal{Q}(\mathcal{K}, p, \mathcal{V})=\exp (2 \mathcal{S}(\mathcal{K}, p, \mathcal{V}))
$$

Remark. The bounds we get are related to a result of Brezis and Merle [BM91] proving the existence of a bound for $\|u\|_{L^{q}\left(\mathbb{R}^{2}, g_{0}\right)}$ for functions $u: \Omega \rightarrow \mathbb{R}$ defined on a bounded domain $\Omega \subset \mathbb{R}^{n}$ satisfying the Kazdan-Warner equation

$$
\Delta_{g_{0}} u=K(x) e^{2 u}
$$

with Dirichlet boundary conditions. Their bound depends on $\left\|K_{g}\right\|_{L^{p}\left(\mathbb{R}^{2}, g_{0}\right)}$ and $\Omega$. The main difference to our results is that Brezis and Merle do not treat functions $u: T^{2} \rightarrow \mathbb{R}$ and that their norms are taken with respect to the flat metric $g_{0}$ whereas we bound osc $u$ in terms of the $L^{p}$-norm with respect to the metric $g=e^{2 u} g_{0}$. 
Now we turn to the proof of Theorem 3.1. The theorem will follow from some propositions and corollaries that will take the rest of this section. For the proof we split the torus $T^{2}$ into three subsets two of which have the property that their fundamental group is mapped trivially to $\pi_{1}\left(T^{2}\right)$. On these two subsets osc $u$ can be estimated using Corollary 3.3 and Proposition 3.6. The third part contains generators of $\pi_{1}\left(T^{2}\right)$ and will be dealt in Proposition 3.8 .

Proposition 3.2. Let $G$ be a bounded open subset of $\mathbb{R}^{2}$ with the standard metric $g_{0}$. For a smooth function $u: \bar{G} \rightarrow \mathbb{R}$ with $u \geq 0,\left.u\right|_{\partial G} \equiv 0$ we set $g=e^{2 u} g_{0}$. We define $\mu_{0}:=\operatorname{area}(G, g)$ and for the Gaussian curvature $K_{g}$ with respect to $g$ let

$$
k(A):=\sup \left\{\int_{\widehat{G}, g} K_{g} \mid \widehat{G} \text { open subset of } G, \operatorname{area}(\widehat{G}, g)=A\right\} .
$$

If there are $\kappa \in] 0,2 \pi[, C>0, r \in] 0,1]$ and $\left.\left.\mu_{1} \in\right] 0, \mu_{0}\right]$, such that

$$
k(A) \leq\left\{\begin{array}{lll}
C \cdot A^{r} \leq \kappa & \text { for } \quad 0 \leq A \leq \mu_{1} \\
\kappa & \text { for } \quad \mu_{1} \leq A \leq \mu_{0}
\end{array}\right.
$$

then

$$
\max u \leq \frac{1}{2}\left|\log \left(1-\frac{\kappa}{2 \pi}\right)\right|+\frac{\kappa}{4 \pi-2 \kappa} \log \left(\frac{\mu_{0}}{\sqrt[r]{r} \mu_{1}}\right)
$$

Before proving this proposition, we will prove a corollary.

COROLLARY 3.3. Suppose the open subset $G$ of $T^{2}$ has the property that any loop $\gamma: S^{1} \rightarrow \bar{G}$ is contractible in $T^{2}$. Let $g_{0}$ be a flat metric on $T^{2}$ and $g=e^{2 u} g_{0}$ another metric on $T^{2}$ with a smooth function $u: T^{2} \rightarrow \mathbb{R}$ satisfying $\left.u\right|_{\partial G} \equiv 0$ and $\left.u\right|_{G} \geq 0$. Suppose for some $\left.p \in\right] 1, \infty[$ we have

$$
\mathcal{K}_{p}=\mathcal{K}_{p}\left(T^{2}, g\right)<4 \pi
$$

Then we get the estimate

$$
\max _{x \in \bar{G}} u(x) \leq \frac{1}{2}\left|\log \left(1-\frac{\mathcal{K}_{p}}{4 \pi}\right)\right|+\frac{\mathcal{K}_{p}}{8 \pi-2 \mathcal{K}_{p}} q \log (2 q)
$$

with $q:=p /(p-1)$.

Proof of Corollary 3.3. Because of the Gauss-Bonnet theorem we have

$$
\left|\int_{\widehat{G}, g} K_{g}\right|=\left|\int_{T^{2}-\widehat{G}, g} K_{g}\right|
$$

for any open subset $\widehat{G} \subset G$. Therefore

$$
\left|\int_{\widehat{G}, g} K_{g}\right| \leq \frac{1}{2} \int_{T^{2}, g}\left|K_{g}\right| \leq \frac{1}{2} \mathcal{K}_{p} .
$$


On the other hand, if we have $\operatorname{area}(\widehat{G}, g)<2^{-q}$ area $\left(T^{2}, g\right)$, then the estimate

$$
\left|\int_{\widehat{G}, g} K_{g}\right| \leq\left\|K_{g}\right\|_{L^{p}(\widehat{G}, g)}(\operatorname{area}(\widehat{G}, g))^{1 / q} \leq \mathcal{K}_{p}\left(\frac{\operatorname{area}(\widehat{G}, g)}{\operatorname{area}\left(T^{2}, g\right)}\right)^{1 / q}
$$

is better.

Since any loop $\gamma: S^{1} \rightarrow \bar{G}$ is contractible in $T^{2}$, we can lift $G$ to $\mathbb{R}^{2}$.

We can apply Proposition 3.2 with $\kappa:=(1 / 2) \mathcal{K}_{p}, r:=1 / q, C:=\mathcal{K}_{p}$ area $\left(T^{2}, g\right)^{-1 / q}$, $\mu_{0}:=\operatorname{area}(G, g) \leq \operatorname{area}\left(T^{2}, g\right)$ and $\mu_{1}:=\min \left\{\mu_{0}, 2^{-q}\right.$ area $\left.\left(T^{2}, g\right)\right\}$.

Proof of Proposition 3.2. For $v \in \mathbb{R}$ we define $G(v):=\{x \in G \mid u(x)>v\}$. The area of $G(v)$ with respect to $g$ (or $g_{0}$ resp.) will be denoted $A(v)$ (or $A_{0}(v)$ resp.). For the length of the boundary $\partial G(v)$ we write $l(v)$ (or $l_{0}(v)$ resp.). The functions $A(v), A_{0}(v), l(v)$ and $l_{0}(v)$ are differentiable at every regular value $v$ of the function $u$. Therefore we get for regular values $v$ :

$$
\begin{aligned}
e^{2 v} A_{0}(v) & \leq A(v) \leq e^{2 \max u} A_{0}(v) \\
e^{2 v} \frac{d}{d v} A_{0}(v) & =\frac{d}{d v} A(v) \\
e^{v} l_{0}(v) & =l(v) \\
\int_{G(v), g} K_{g} & \leq k(A(v)) .
\end{aligned}
$$

On the other hand, according to Lemma 2.1 we have

$$
\int_{G(v), g} K_{g}=\int_{G(v), g} \Delta_{g} u=-\int_{\partial G(v)} * d u=\int_{\partial G(v), g_{0}}|d u|_{g_{0}} .
$$

The last equation follows since $u$ is equal to $v$ on $\partial G(v)$ and greater than $v$ on $G(v)$ and therefore $* d u$ is negatively oriented on $\partial G(v)$. We also see that $\int_{G(v), g} K_{g}$ is positive for every regular value $v \in u(G)$ of the function $u$.

Using (6) and (7) we calculate

$$
\begin{aligned}
-\frac{d}{d v} A_{0}(v) & =\int_{\partial G(v), g_{0}} \frac{1}{|d u|_{g_{0}}} \geq \frac{l_{0}(v)^{2}}{\int_{\partial G(v), g_{0}}|d u|_{g_{0}}} \\
& \geq \frac{l_{0}(v)^{2}}{k(A(v))}
\end{aligned}
$$

for any regular value $v \in u(G)$ of $u$. Now we apply the isoperimetric inequality

$$
l_{0}(v)^{2} \geq 4 \pi A_{0}(v)
$$


and we get

$$
-\frac{d}{d v} A_{0}(v) \geq \frac{4 \pi}{k(A(v))} A_{0}(v)
$$

We set $\mu_{2}:=\sqrt[r]{r} \mu_{1}$ and $u_{2}:=\inf \left\{\alpha \in\left[0, \max u\left[\mid A(\alpha) \leq \mu_{2}\right\}\right.\right.$. Let us distinguish between the two cases $v \geq u_{2}$ and $v<u_{2}$.

We start with $v \geq u_{2}$. In this case the inequalities (3), (田), (10) and the bound of $k(A)$ provide the estimate

$$
-\frac{d}{d v} A(v) \geq \frac{4 \pi}{C \cdot A(v)^{r}} e^{2(v-\max u)} A(v)
$$

and therefore

$$
-\frac{1}{r} \frac{d}{d v}\left(A(v)^{r}\right) \geq \frac{4 \pi}{C} e^{2(v-\max u)} .
$$

We use the following lemma. Note that any monotonically increasing function is differentiable almost everywhere.

LEMMA 3.4. Let $f, g:[a, b] \rightarrow \mathbb{R}$ be functions with $f$ monotonically increasing, $g$ continuously differentiable and $f(a)=g(a)$. If $f^{\prime} \geq g^{\prime}$ almost everywhere, then $f(b) \geq g(b)$.

This lemma ensures that we can integrate the previous inequality from $u_{2}$ to $\max u$. We use $A(\max u)=0$ and get

$$
\frac{1}{r} A\left(u_{2}\right)^{r} \geq \frac{2 \pi}{C} e^{-2 \max u}\left(e^{2 \max u}-e^{2 u_{2}}\right)=\frac{2 \pi}{C}\left(1-e^{2\left(u_{2}-\max u\right)}\right) .
$$

Furthermore $A\left(u_{2}\right)=\lim _{\alpha u_{2}} A(\alpha) \leq \mu_{2}=\sqrt[r]{r} \mu_{1}$ and $C \mu_{1}{ }^{r} \leq \kappa$ yield

$$
1-e^{2\left(u_{2}-\max u\right)} \leq \frac{C}{2 \pi r} A\left(u_{2}\right)^{r} \leq \frac{\kappa}{2 \pi}
$$

and therefore

$$
(\max u)-u_{2} \leq \frac{1}{2}\left|\log \left(1-\frac{\kappa}{2 \pi}\right)\right|
$$

Now we treat the case $v<u_{2}$. From the estimate (11) we know for small $\varepsilon>0$

$$
A_{0}\left(u_{2}-\varepsilon\right) \geq e^{-2 \max u} A\left(u_{2}-\varepsilon\right) \geq e^{-2 \max u} \mu_{2} \geq e^{-2 u_{2}} \mu_{3},
$$

with

$$
\mu_{3}:=\left(1-\frac{\kappa}{2 \pi}\right) \mu_{2} .
$$


Using (4) we obtain

$$
A_{0}(v) \geq \int_{v}^{u_{2}-\varepsilon} e^{-2 \bar{v}}\left(-\frac{d}{d \bar{v}} A(\bar{v})\right) d \bar{v}+e^{-2 u_{2}} \mu_{3} .
$$

Here we used the fact that any monotonically decreasing function $h:[a, b] \rightarrow \mathbb{R}$ satisfies $h(a)-h(b) \geq \int_{a}^{b}-h^{\prime}(t) d t$. In particular we can integrate over the singularities. Inequality (10) then provides

$$
-\frac{d}{d v} A(v) \geq \frac{4 \pi}{\kappa} e^{2 v}\left(\int_{v}^{u_{2}-\varepsilon} e^{-2 \bar{v}}\left(-\frac{d}{d \bar{v}} A(\bar{v})\right) d \bar{v}+e^{-2 u_{2}} \mu_{3}\right) .
$$

Let $f$ be the solution of the integral equality corresponding to this integral inequality, i.e.

$$
f(v)=\frac{4 \pi}{\kappa} e^{2 v}\left(\int_{v}^{u_{2}-\varepsilon} e^{-2 \bar{v}} f(\bar{v}) d \bar{v}+e^{-2 u_{2}} \mu_{3}\right) .
$$

Via differentiation we get the differential equation

$$
\frac{d}{d v} f(v)=2 f(v)-\frac{4 \pi}{\kappa} f(v)
$$

with initial value $f\left(u_{2}-\varepsilon\right)=(4 \pi / \kappa) e^{-2 \varepsilon} \mu_{3}$. So the solution is

$$
f(v)=\frac{4 \pi}{\kappa} e^{-2 \varepsilon} \mu_{3} e^{\left(\frac{4 \pi}{\kappa}-2\right)\left(u_{2}-v-\varepsilon\right)} .
$$

Here we use another elementary lemma.

LEMMA 3.5. Let $f_{1}$ and $f_{2}$ be $L^{1}$-functions on $[a, b]$ and $g_{1}, g_{2}:[a, b] \rightarrow \mathbb{R}^{+}$continuous functions. Let $C \in \mathbb{R}^{+}$. We assume that for any $t \in[a, b]$ we have

$$
\begin{aligned}
& f_{1}(t) \geq g_{1}(t)\left(C+\int_{a}^{t} g_{2}(s) f_{1}(s) d s\right) \\
& f_{2}(t)=g_{1}(t)\left(C+\int_{a}^{t} g_{2}(s) f_{2}(s) d s\right) .
\end{aligned}
$$

Then we get $f_{1}(t) \geq f_{2}(t)$ for any $t \in[a, b]$.

Thus we obtain

$$
-\frac{d}{d v} A(v) \geq f(v)=\frac{4 \pi}{\kappa} \mu_{3} e^{-2 \varepsilon} e^{\left(\frac{4 \pi}{\kappa}-2\right)\left(u_{2}-v-\varepsilon\right)}
$$


and the limit $\varepsilon \rightarrow 0$ yields

$$
-\frac{d}{d v} A(v) \geq f(v)=\frac{4 \pi}{\kappa} \mu_{3} e^{\left(\frac{4 \pi}{\kappa}-2\right)\left(u_{2}-v\right)}
$$

Now integration from 0 to $u_{2}$ using Lemma 3.4 yields

$$
\mu_{0}-\mu_{2} \geq A(0)-A\left(u_{2}\right) \geq \mu_{3}\left(1-\frac{\kappa}{2 \pi}\right)^{-1}\left(e^{\left(\frac{4 \pi}{\kappa}-2\right) u_{2}}-1\right) .
$$

Hence

$$
\begin{aligned}
u_{2} & \leq \frac{\kappa}{4 \pi-2 \kappa} \log \left(1+\left(1-\frac{\kappa}{2 \pi}\right) \frac{\left(\mu_{0}-\mu_{2}\right)}{\mu_{3}}\right) \\
& =\frac{\kappa}{4 \pi-2 \kappa} \log \left(\frac{\mu_{0}}{\sqrt[r]{r} \mu_{1}}\right) .
\end{aligned}
$$

This together with (11) yields the estimate of the proposition.

Proposition 3.6. Let $u: \bar{G} \rightarrow \mathbb{R}, u \leq 0$ be a smooth function on the bounded open subset $G \subset \mathbb{R}^{2}$ satisfying the boundary condition $\left.u\right|_{\partial G} \equiv 0$. Let $g_{0}$ be the restriction of the standard metric on $\mathbb{R}^{2}$. We set $g:=e^{2 u} g_{0}$. For $\left.p \in\right] 1, \infty[$ we define $q:=p /(p-1)$. Then

$$
\min u \geq-\frac{q \mathcal{K}_{p}^{-}(G, g)}{4 \pi}
$$

Proof of Proposition 3.6. This time we define $\kappa:=\mathcal{K}_{p}^{-}(G, g)$ and $G(v):=\{x \in$ $G \mid u(x)<v\}$. As in the proof of Proposition 3.2 let $A(v)$ and $l(v)$ or $A_{0}(v)$ and $l_{0}(v)$ resp. be the area of $G(v)$ and the length of $\partial G(v)$ with respect to $g$ or $g_{0}$ resp. Again we have (4), (5) and (9) whereas we have to modify (3) and (4):

$$
\begin{aligned}
e^{2 v} A_{0}(v) & \geq A(v) \\
\int_{G(v), g} K_{g} & =-\int_{\partial G(v), g_{0}}|d u|_{g_{0}} .
\end{aligned}
$$

Furthermore we get

$$
k(v):=-\int_{G(v), g} K_{g} \leq\left\|K_{g}^{-}\right\|_{L^{p}(G(v)), g} \operatorname{area}(G(v), g)^{1 / q} \leq \kappa\left(\frac{A(v)}{A(0)}\right)^{1 / q} .
$$

Inequality (8) holds with a different sign:

$$
\frac{d}{d v} A_{0}(v) \geq \frac{l_{0}(v)^{2}}{k(v)}
$$


Together with (9) and (13) this yields

$$
\frac{d}{d v} A_{0}(v) \geq \frac{4 \pi}{\kappa} A_{0}(v)\left(\frac{A(0)}{A(v)}\right)^{1 / q} .
$$

Finally with (4) and (12) we obtain

$$
\frac{d}{d v} A(v) \geq \frac{4 \pi}{\kappa} A(v)\left(\frac{A(0)}{A(v)}\right)^{1 / q}
$$

We use again Lemma 3.4 in order to integrate this inequality from min $u$ to 0 , and we get

$$
q\left(A(0)^{1 / q}-A(\min u)^{1 / q}\right) \geq \frac{4 \pi}{\kappa} A(0)^{1 / q}|\min u| .
$$

Since $A(\min u)=0$ this implies

$$
|\min u| \leq \frac{q \kappa}{4 \pi}
$$

LEMMA 3.7. Let $G$ be an open set in $\left(T^{2}, g\right)$ with smooth boundary. We suppose that there are closed curves $c_{1}:[0,1] \rightarrow G$ and $c_{2}:[0,1] \rightarrow T^{2}-\bar{G}$, whose corresponding homology classes in $H_{1}\left(T^{2}, \mathbb{Z}\right)$ are not zero. Then

$$
\operatorname{length}_{g}(\partial G) \geq 2 \operatorname{sys}_{1}\left(T^{2}, g\right) \text {. }
$$

Proof. If we regard $\bar{G}$ as a 2 -cycle, then clearly $\partial G$ is homologous to zero. Now decompose $\partial G$ into its components. Each component is diffeomorphic to $S^{1}$.

We will show that there is at least one component non-homologous to zero. Together with $[\partial G]=0$ this implies that are at least two such components and therefore we get the statement of the lemma.

So let us suppose that all components of $\partial G$ are homologous to zero. Let $\pi: \mathbb{R}^{2} \rightarrow T^{2}$ be the universal covering. Then $\pi^{-1}(\partial G)$ is diffeomorphic to a disjoint union of countably many $S^{1}$. We write

$$
\pi^{-1}(\partial G)=\bigcup_{i \in \mathbb{N}}^{\cdot} Y_{i}
$$

with $Y_{i} \cong S^{1}$. We choose lifts $\tilde{c}_{i}: \mathbb{R} \rightarrow \mathbb{R}^{2}$ of $c_{i}$, i. e. $\pi\left(\tilde{c}_{i}(t+z)\right)=c_{i}(t)$ for all $t \in[0,1], z \in \mathbb{Z}$ and $i=1,2$. Then we take a path $\tilde{\gamma}:[0,1] \rightarrow \mathbb{R}^{2}$ joining $\tilde{c}_{1}(0)$ to $\tilde{c}_{2}(0)$. We define $I$ to be the set of all $i \in \mathbb{N}$ such that $Y_{i}$ meets the trace of $\tilde{\gamma}$. The set $I$ is finite. Using the Theorem of Jordan and Schoenfliess about simply closed curves in $\mathbb{R}^{2}$ we can inductively construct a compact set $K \subset \mathbb{R}^{2}$ with boundary $\bigcup_{i \in I} Y_{i}$. Either $\tilde{c}_{1}(0)$ or $\tilde{c}_{2}(0)$ is in the interior of $K$. But if $\tilde{c}_{i}(0)$ is in the interior of $K$, then the whole trace $\tilde{c}_{i}(\mathbb{R})$ is contained in $K$. Furthermore, $\tilde{c}_{i}(\mathbb{R})=\pi^{-1}\left(c_{i}([0,1])\right)$ is closed and therefore compact. This implies that $c_{i}$ is homologous to zero in contradiction to our assumption. 
Proposition 3.8. Suppose that $T^{2}$ carries a Riemannian metric $g=e^{2 u} g_{0}$ with $g_{0}$ flat. Let $c_{i}: S^{1} \rightarrow T^{2}, i=1,2$ be closed paths non-homologous to zero. We set

$$
v_{1}:=\max _{t \in S^{1}} u \circ c_{1}(t) \quad \text { and } \quad v_{2}:=\min _{t \in S^{1}} u \circ c_{2}(t) .
$$

Then

$$
\begin{aligned}
& v_{2}-v_{1} \leq \frac{\mathcal{K}_{1}\left(T^{2}, g\right) \mathcal{V}\left(T^{2}, g_{0}\right)}{8}, \\
& v_{2}-v_{1} \leq \frac{\mathcal{K}_{1}\left(T^{2}, g\right) \mathcal{V}\left(T^{2}, g\right)}{8} .
\end{aligned}
$$

Proof. The statement is void for $v_{2} \leq v_{1}$, therefore we can assume $v_{2}>v_{1}$. We set $\mathcal{K}_{1}:=\mathcal{K}_{1}\left(T^{2}, g\right)$. Let $\left.v \in\right] v_{1}, v_{2}[$ be a regular value of $u$. As in the proof of the previous proposition we set $G(v):=\left\{x \in T^{2} \mid u(x)<v\right\}$, let $A(v)$ be the area $G(v)$ with respect to $g, l(v)$ the length of $\partial G(v)$ with respect to $g$, and $A_{0}(v)$ and $l_{0}(v)$ the area and length with respect to $g_{0}$. In analogy to (7) we get

$$
\int_{G(v), g} K_{g}=-\int_{T^{2}-G(v), g} K_{g}=-\int_{\partial G(v)} * d u
$$

and therefore

$$
\int_{\partial G(v), g_{0}}|d u|_{g_{0}}=\int_{\partial G(v), g}|d u|_{g}=\int_{\partial G(v)} * d u \leq \frac{1}{2} \int_{T^{2}, g}\left|K_{g}\right| \leq \frac{\mathcal{K}_{1}}{2} .
$$

We obtain

$$
\frac{d}{d v} A_{0}(v)=\int_{\partial G(v), g_{0}} \frac{1}{|d u|_{g_{0}}} \geq \frac{l_{0}(v)^{2}}{\int_{\partial G(v), g_{0}}|d u|_{g_{0}}} \geq 2 \frac{l_{0}(v)^{2}}{\mathcal{K}_{1}} .
$$

Using Lemma 3.7 we know that the right hand side of this inequality is greater than or equal to $8 \operatorname{sys}_{1}\left(T^{2}, g_{0}\right)^{2} / \mathcal{K}_{1}$.

Integration yields

$$
\operatorname{area}\left(T^{2}, g_{0}\right) \geq A_{0}\left(v_{2}\right)-A_{0}\left(v_{1}\right) \geq 8 \frac{\operatorname{sys}_{1}\left(T^{2}, g_{0}\right)^{2}}{\mathcal{K}_{1}}\left(v_{2}-v_{1}\right),
$$

and therefore the statement of (a).

Similarly, we show statement (b):

$$
\begin{aligned}
& \frac{d}{d v} A(v)=\int_{\partial G(v), g} \frac{1}{|d u|_{g}} \geq \frac{l(v)^{2}}{\int_{\partial G(v), g}|d u|_{g}} \geq 8 \frac{\operatorname{sys}_{1}\left(T^{2}, g\right)^{2}}{\mathcal{K}_{1}}, \\
& \operatorname{area}\left(T^{2}, g\right) \geq A\left(v_{2}\right)-A\left(v_{1}\right) \geq 8 \frac{\operatorname{sys}_{1}\left(T^{2}, g\right)^{2}}{\mathcal{K}_{1}}\left(v_{2}-v_{1}\right) .
\end{aligned}
$$


An alternative way to prove (b) is to use (a) together with Lemma 2.2 .

Proof of Theorem 3.1. Again we set $G(v):=\left\{x \in T^{2} \mid u(x)>v\right\}$. Let $v_{2}$ be the supremum of all $v \in \mathbb{R}$ with the property that there is a closed path $c_{2}(v)$ : $S^{1} \rightarrow G(v)$ that is non-homologous to zero in $T^{2}$. Similarly, we define $\widehat{G}(v):=\{x \in$ $\left.T^{2} \mid u(x)<v\right\}$, and let $v_{1}$ be the infimum of all $v \in \mathbb{R}$ for which there is a closed path $c_{1}(v): S^{1} \rightarrow \widehat{G}(v)$ that is non-homologous to zero in $T^{2}$.

For any $\varepsilon>0$ we have $\max \left(u \circ c_{1}\left(v_{1}+\varepsilon\right)\right)<v_{1}+\varepsilon$ and $\min \left(u \circ c_{2}\left(v_{2}-\varepsilon\right)\right)>v_{2}-\varepsilon$. We apply Proposition 3.8, and the limes $\varepsilon \rightarrow 0$ yields

$$
\begin{aligned}
v_{2}-v_{1} & \leq \frac{\mathcal{K}_{1}\left(T^{2}, g\right) \mathcal{V}\left(T^{2}, g_{0}\right)}{8} \\
& \leq \frac{\mathcal{K}_{p}\left(T^{2}, g\right) \mathcal{V}\left(T^{2}, g_{0}\right)}{8}
\end{aligned}
$$

Now we apply Corollary 3.3 for $G:=G(v)$ and $v:=v_{2}+\varepsilon$ where we replace $u$ by $u-v$. We get in the limit $\varepsilon \rightarrow 0$

$$
(\max u)-v_{2} \leq \frac{1}{2}\left|\log \left(1-\frac{\mathcal{K}_{p}}{4 \pi}\right)\right|+\frac{\mathcal{K}_{p}}{8 \pi-2 \mathcal{K}_{p}} q \log (2 q),
$$

with $q:=p /(p-1)$.

Similarly, Proposition 3.6 yields

$$
v_{1}-(\min u) \leq \frac{q \mathcal{K}_{p}(G, g)}{4 \pi} .
$$

Adding inequalities (15), (16) and (17) we obtain statement (a).

The proof of statement (b) is completely analogous.

\section{An estimate on the disk}

From the results of the previous section is not difficult to derive another theorem.

THEOREM 4.1. Let $g$ be a Riemannian metric on a domain $G$ whose closure is diffeomorphic to the 2-dimensional disk. We write $g$ as $g=e^{2 u} g_{0}$ with $g_{0}$ flat and $\left.u\right|_{\partial G} \equiv 0$. For $\left.p \in\right] 1, \infty\left[\right.$ we assume $\mathcal{K}_{p}^{+}:=\mathcal{K}_{p}^{+}(G, g)<2 \pi$. Then we get the estimate

$$
\max u \leq \frac{1}{2}\left|\log \left(1-\frac{\mathcal{K}_{p}^{+}}{2 \pi}\right)\right|+\frac{\mathcal{K}_{p}^{+}}{4 \pi-2 \mathcal{K}_{p}^{+}} q \log q
$$

with $q:=p /(p-1)$. 
Proof of Theorem 4.1. W.l.o.g. we can assume $u \geq 0$.

For any open subset $\widehat{G} \subset G$ we have

$$
\begin{aligned}
\int_{\widehat{G}, g} K_{g} & \leq\left\|K_{g}^{+}\right\|_{L^{1}(\widehat{G}, g)} \\
& \leq\left\|K_{g}^{+}\right\|_{L^{p}(\widehat{G}, g)} \operatorname{area}(\widehat{G}, g)^{1 / q} \leq \mathcal{K}_{p}^{+}\left(\frac{\operatorname{area}(\widehat{G}, g)}{\operatorname{area}(G, g)}\right)^{1 / q} .
\end{aligned}
$$

We can apply Proposition 3.2 with $\kappa=\mathcal{K}_{p}^{+}, r=1 / q, C=\mathcal{K}_{p}^{+}$area $(G, g)^{-1 / q}$ and $\mu_{0}=\mu_{1}=\operatorname{area}(G, g)$ and we directly get the theorem.

\section{Cylindrical and conical examples}

In the previous section we gave a bound on osc $u$ in terms of the $L^{p}$-norm of the Gaussian curvature $K, p>1$, the area and the systole. Now we will give some examples showing that osc $u$ is not bounded by a function of the $L^{1}$-norm of $K$, the area and the systole.

In contrast to this, note that the diameter of $T^{2}$ is bounded by a function depending on $\int_{T^{2}}\left|K_{g}\right|$, area $\left(T^{2}, g\right)$ and and $\operatorname{sys}_{1}\left(T^{2}, g\right)$, provided that $\int_{T^{2}}\left|K_{g}\right|<4 \pi$ Amm98, Korollar 3.6.8].

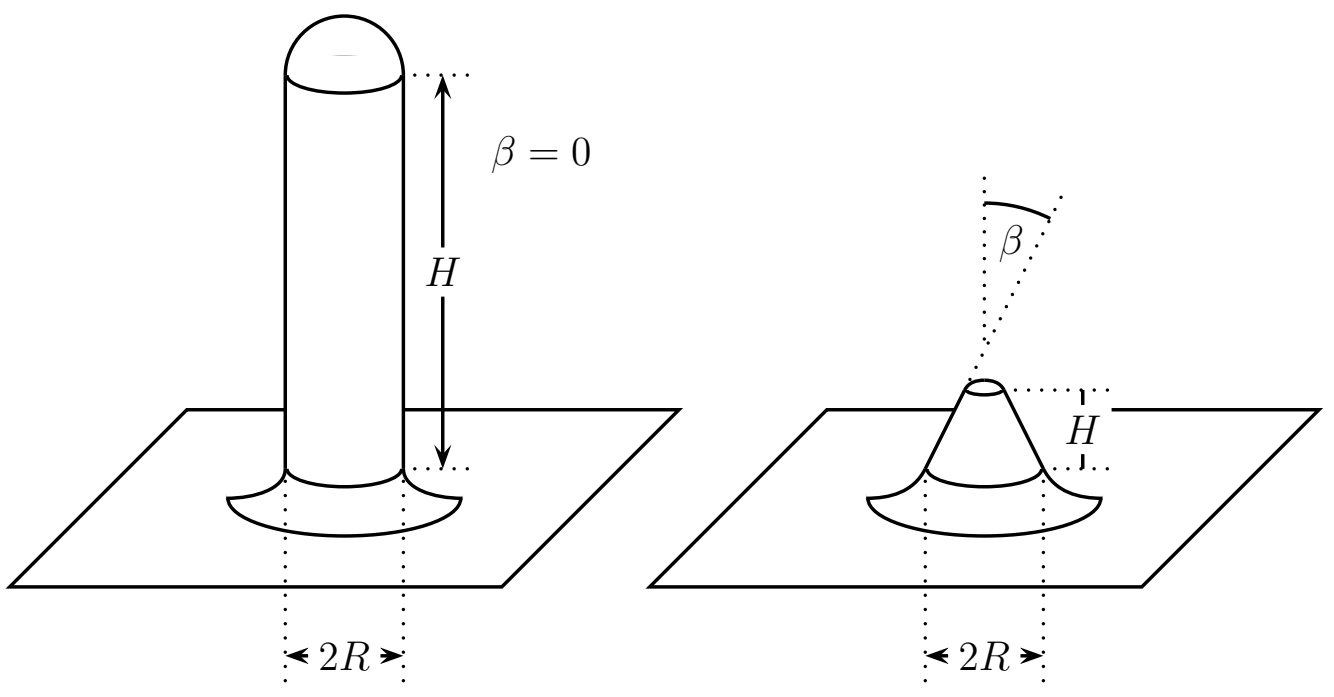

Figure 2: cylindrical and conical metric 
In order to discuss the properties of our examples we will use a lemma that can easily be proven by using Lemma 2.1 and the Gauss-Bonnet theorem.

LEMMA 5.1. Assume that a disk D carries a rotationally symmetric Riemannian metric $g$ and that in a neighborhood of the boundary $g$ is isometric to a flat ring of the form $\left(B_{R}(0)-B_{r}(0) \subset \mathbb{R}^{2}, g_{\text {eucl }}\right)$. Then there is a rotationally symmetric smooth function $u: B_{R}(0) \rightarrow \mathbb{R}$ vanishing in a neighborhood of the boundary such that $(D, g)$ is isometric to $\left(B_{R}(0), e^{2 u} g_{\text {eucl }}\right)$. The function $u$ is uniquely determined by these properties.

The idea behind the construction of the metrics in this section is to start with a flat torus $\left(T^{2}, g_{0}\right)$, to cut out a flat round disk and to replace it by a disk $D^{\prime}$ with a rotationally symmetric metric $g^{\prime}$. Because of the preceeding lemma the metric $g$ obtained by this replacement can be written as $e^{2 u} g_{0}$ where $u$ is a smooth function supported on the disk. Viewed as a function on the disk, $u$ is rotationally symmetric. Therefore osc $u$ can be easily estimated using polar coordinates.

The disks $\left(D^{\prime}, g^{\prime}\right)$ we glue in are described by figure 2. For the cylindrical metric we construct $\left(D^{\prime}, g^{\prime}\right)$ as follows: we take a cylinder of height $H$ and radius $R$, glue it together with a half sphere of radius $R$ at one end and a suitable socket on the other end. After smoothing we get $\left(D^{\prime}, g^{\prime}\right)$. The resulting metric on $T^{2}$, the cylindrical metric, will be denoted $g_{R, H, 0}$.

Similarly, for the conical metric $g_{R, H, \beta}$ (Figures 2 and 3) we take a truncated cone of height $H$, opening angle $\beta>0$ and the two boundary components are circles of radius $R$ and $\rho=R-H \sin \beta$. The end of the truncated cone corresponding to $\rho$ is closed smoothly by a topological disk and the other end is put on a socket.

Now we write $g^{\prime}=e^{2 u} g_{0}$ and express $u$ in geodesic polar coordinates centered at the center of $D^{\prime}$. On the cylinder or cone resp. $u$ is harmonic and therefore has the form

$$
u(r, \varphi)=a+b \log r
$$

The Gauss-Bonnet theorem yields $b=\sin \beta-1$. An elementary calculation shows

$$
\operatorname{osc} u \geq\left(\frac{1}{\sin \beta}-1\right) \log \left(\frac{R}{\rho}\right)
$$

for the conical metric and

$$
\operatorname{osc} u \geq H / R
$$

for the cylindrical metric.

Using Gauss-Bonnet we also get

$$
\int_{T^{2}}\left|K_{g_{R, H, \beta}}\right|=4 \pi(1-\sin \beta) .
$$




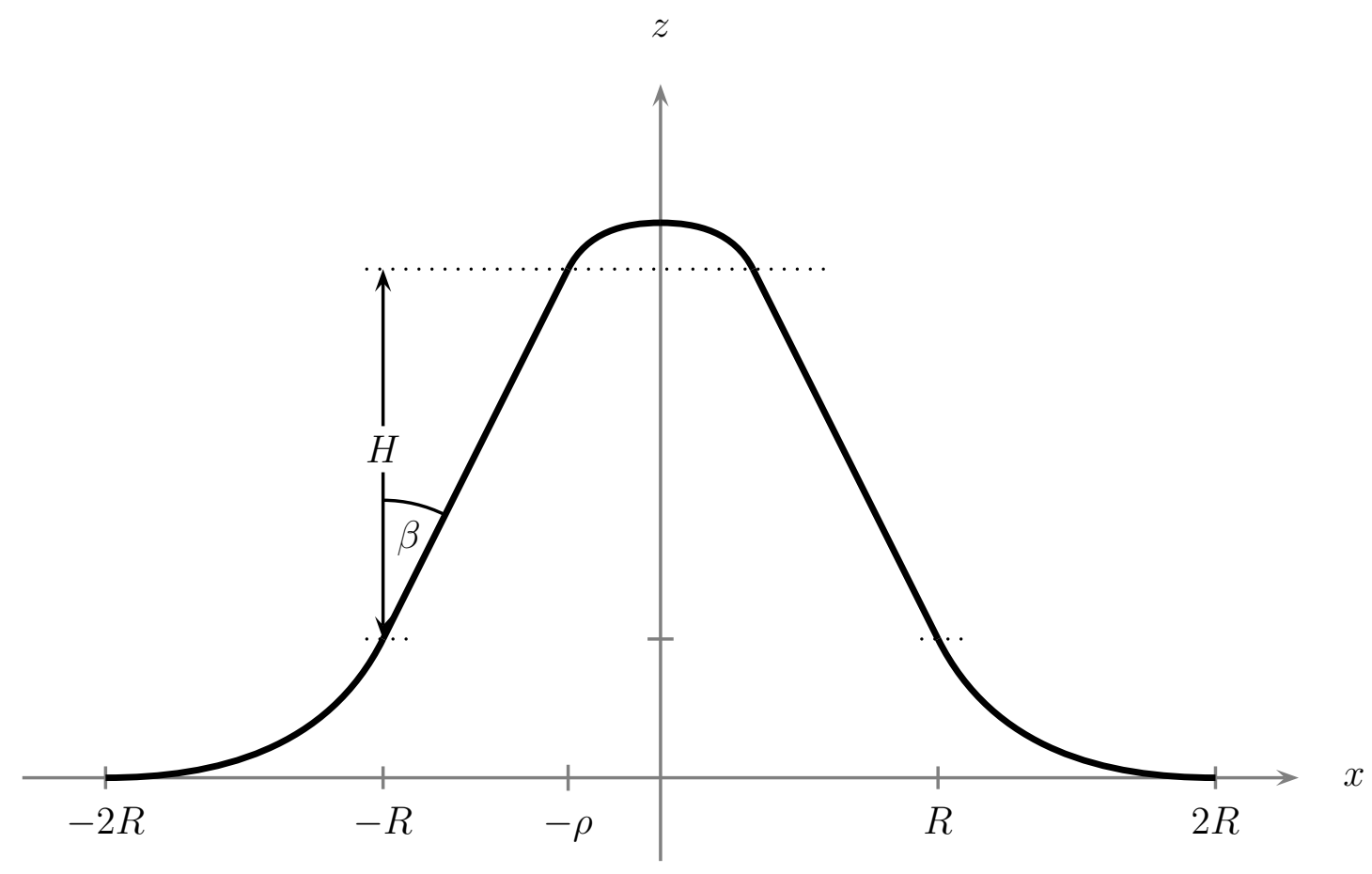

Figure 3: The conical metric

If $R$ is sufficiently small, the systole does not depend on $H, R$ and $\beta$.

Now for fixed $\beta \geq 0$ choose sequences $H_{i}$ and $R_{i}$ such that area $\left(T^{2}, g_{R_{i}, H_{i}, \beta}\right)$ is constant and such that $H_{i} / R_{i} \rightarrow \infty$ or $\rho_{i} / R_{i} \rightarrow 0$ resp.

So we have constructed families of metrics $g_{R_{i}, H_{i}, \beta}$ on $T^{2}$ with fixed $\int|K|$, fixed area and fixed systole but osc $u_{i} \rightarrow \infty$. 


\section{References}

[Amm] B. Ammann, Spectral estimates on 2-tori, Preprint in preparation.

[Amm98] B. Ammann, Spin-Strukturen und das Spektrum des Dirac-Operators, Ph.D. thesis, University of Freiburg, Germany, 1998, Shaker-Verlag Aachen 1998.

[Bär98] C. Bär, Extrinsic bounds for eigenvalues of the Dirac operator, Ann. Global Anal. Geom. 16 (1998), no. 6, 573-596.

[Bes87] A. L. Besse, Einstein manifolds, Ergebnisse der Mathematik und ihrer Grenzgebiete, 3. Folge, no. 10, Springer-Verlag, 1987.

[BM91] H. Brezis and F. Merle, Uniform estimates and blow-up behavior for solutions of $-\Delta u=V(x) e^{u}$ in two dimensions, Comm. Partial Diff. Equat. 16 (1991), no. 8 \& 9, 1223-1253.

[Che81] Bang-Yen Chen, On the total curvature of immersed manifolds. V. Csurfaces in Euclidean m-space, Bull. Inst. Math. Acad. Sinica 9 (1981), no. $4,509-516$.

[Che98] Xiuxiong Chen, Weak limits of Riemannian metrics in surfaces with integral curvature bound, Calc. Var. Partial Differential Equations 6 (1998), no. 3, 189-226.

[Gro81] M. Gromov, Structures métriques pour les variétés Riemanniennes, CEDIC, Paris, 1981.

[LS84] J. Langer and D. Singer, Curves in the hyperbolic plane and mean curvature of tori in 3-space, Bull. London Math. Soc. 16 (1984), 531-534.

[LY82] P. Li and S.T. Yau, A new conformal invariant and its applications to the Willmore Conjecture and the first eigenvalue of compact surfaces, Invent. Math. 69 (1982), 269-291.

[MR85] S. Montiel and A. Ros, Minimal immersions of surfaces by the first eigenfunctions and conformal area, Invent. Math. 83 (1985), no. 1, 153-166.

[Pin85] U. Pinkall, Hopf tori in $S^{3}$, Invent. Math. 81 (1985), no. 2, 379-386.

[Ros97] A. Ros, The Willmore conjecture in the real projective space, Preprint, 1997.

[Sim93] L. Simon, Existence of surfaces minimizing the Willmore functional, Comm. Anal. Geom. 1 (1993), no. No. 2, 281-326. 
[ST70] K. Shiohama and R. Takagi, A characterization of a standard torus in $E^{3}$, J. Diff. Geom. 4 (1970), 477-485.

[Tho23] G. Thomsen, Über konforme Geometrie, I: Grundlagen der konformen Flächentheorie, Abh. Math. Sem. Hamburg 3 (1923), 31-56.

[Top98] P. Topping, Towards the Willmore conjecture, Preprint, 1998.

[Wei78] J. L. Weiner, On a problem of Chen, Willmore, et al., Ind. Math. J. 27 (1978), no. 1, 19-35.

[Wil65] T. J. Willmore, Note on embedded surfaces, An. Şti. Univ. "Al. I. Cuza" Iaşi Secţ. I a Mat. (N.S.) 11B (1965), 493-496.

[Wil71] T. J. Willmore, Mean curvature of Riemannian immersions, J. London Math. Soc. (2) 3 (1971), 307-310.

Bernd Ammann

Mathematisches Institut

Universität Freiburg

Eckerstr. 1

79104 Freiburg

Germany

ammann@mathematik. uni-freiburg. de 